\title{
Design and Analysis of Chinese-Korean Translation System Based on Deep Transfer Learning
}

\author{
Jun Xu \\ The Department of Korean Studies, Dalian University of Foreign Languages, Dalian 116044, China \\ Correspondence should be addressed to Jun Xu; xujun@dlufl.edu.cn
}

Received 13 December 2021; Revised 6 January 2022; Accepted 13 January 2022; Published 7 February 2022

Academic Editor: Hangjun Che

Copyright (C 2022 Jun Xu. This is an open access article distributed under the Creative Commons Attribution License, which permits unrestricted use, distribution, and reproduction in any medium, provided the original work is properly cited.

\begin{abstract}
In order to better establish the Chinese-Korean translation system model, the deep transfer learning and the model system are tested and analyzed, and the following analysis results are obtained. By discussing the different adjustment mechanisms of deep transfer learning under MMD metric and Wasserstein metric, we can see that, in the MMD metric model, through analyzing datasets 1 and 2, the highest accuracy rate is $83.1 \%$ under multisource weight adjustment mechanism under MMD metric and the lower accuracy rate is $62.7 \%$ under no weight adjustment mechanism, and the accuracy rates of datasets 1 and 2 are higher than the average. Under Wasserstein metric, the accuracy of dataset 1 is $82.5 \%$ under multisource weight and $68.5 \%$ under no source weight, both of which are higher than the average. Three EEGNet models, EEGNet_0, EEGNet_1, and EEGNet_2, were established for comparative testing; according to the test results, it can be seen that EEGNet_1 has high accuracy and can be preferred for system establishment. By comparing the Chinese-Korean translation model with the blockchain model and the traditional translation model, it can be seen that when the translation sentences are 100 sentences, the average response time and peak traffic response time of the Chinese-Korean translation model are lower than those of the traditional translation model and the test conclusion is passed. When the test sentences are 1000 sentences, the average response time and peak traffic corresponding time of the Chinese-Korean translation model are still lower than those of the traditional method. Therefore, it can be seen that the efficiency and winning rate of the Chinese-Korean translation model are higher than those of the traditional translation system and meet the needs. According to the analysis of the performance test results of the translation system, it can be seen that the average response time and success rate of the Chinese and Korean translation system under different data are higher than those of the traditional translation system. When the test data are 500, the average response time of the translation system is $13 \mathrm{~ms}$ and the accuracy rate is $100 \%$. When the test data are 3000 , the average response time is $99 \mathrm{~ms}$ and the success rate is $99.6 \%$. Therefore, the success rate of the translation system is basically above $99.6 \%$, which is higher than that of the traditional translation system. In contrast, the Chinese-Korean translation system can improve the translation efficiency and accuracy and can be preferred.
\end{abstract}

\section{Introduction}

Through the model building and comparison of deep transfer learning, we choose the best transfer learning method to support the Chinese-Korean translation system to achieve higher accuracy and efficiency. By establishing the Chinese-Korean translation model systematically, comparing the system with the blockchain system and traditional translation system, it is known that the translation system has higher accuracy and efficiency.

Literature [1] combines nonlinear photons, traditional black box deep learning, and deep learning integral equation through a new method, i.e., deep learning method. It can be used to prove whether artificial intelligence can learn nonlinearly. Experiments show that deep learning network can simulate training to judge the abnormal position of moving objects. Literature [2] trains deep learning network through a large amount of training and data to promote CNN's progress in related fields. Literature [3] realizes NF scale-out and load balancing by flexibly migrating related traffic. In this way, the challenges related to minimizing service resources are solved. Through GNN and DRL to further improve QOS and reduce the delay of deep migration, compared with its most advanced technology, the 
required time is increased by $71.6 \%$. In order to solve the problem of extracting biological information from a large amount of weather radar data, literature [4] studies the deep learning method. Two methods, rendering and mapping, are used to segment the convolution network image and reveal the intensity and depth of the migration pattern. Literature [5] studies the recognition of vehicle-related information by studying deep migration and studies ImageNet data under VGG-19 in the same space. The research shows that its accuracy is as high as $97.73 \%$, which solves a series of problems caused by insufficient samples, and it has high efficiency. Literature [6] seismic imaging is an active research field recently, and this method is used to solve the problem of map imaging. At present, the least square method is the most advanced method for seismic structure acquisition. The depth migration convolution method, which combines Hessian with least square migration method, can provide higher quality images than traditional methods. Literature [7] proposes the Faster R-CNN algorithm in order to improve the high similarity of colors in complex background and reduce the efficiency reduction caused by occlusion. Through relevant experiments, it is proved that transplanting the training model into the system can accurately improve the detection accuracy. It lays a foundation for the research of automatic picking device. Literature [8] uses the Faster R-CNN method to improve the efficiency of collecting and recognizing the characteristic part of TCM tooth pattern tongue. Combining Faster-CNN with the fine-tune model, the experimental results show that the model is not affected by the location of pathological changes in images, has strong adaptability, and can well complete the task of local feature recognition and improve the recognition efficiency. Literature [9] aims to alleviate the problem that the depth learning DL model can effectively record and reconstruct underground velocity images, but the knowledge of adjoint operators will be discarded, resulting in poor reconstruction quality. This paper introduces the development of a DLFWI method, which improves the reconstruction speed and high resolution method, and at the same time, its antinoise ability is greatly enhanced. Literature [10] solves the problem of difficult recognition of fruit image, color, size, and other features in the process of automatic picking by developing a deep migration fruit image recognition method. The research shows that, by introducing this deep transfer learning method, the recognition accuracy is greatly improved and the accuracy is increased to 99\%. Translation needs to be in multiple dimensions [11], and a common problem needs to be transformed into a different form. Through the OntoMorph method, the syntax is transformed. OntoMorph has become the core of communication translation. Literature [12] translates multiple languages through the NMT single neural machine translation model. According to its research results, it can translate the multilingual model of up to 12 languages and improve the translation quality. At the same time, it shows us some interesting cases in mixed language translation. The EBMT translation model [13] has been proved to be successful in translation function by experimental research. Through EBMT, the "low-density" language is reduced to ensure its translation quality. The translation database is accurately matched, so that its translation is unlimited, and the amount of text required for translation and the time required for translation are reduced. Literature [14] develops cell-free protein synthesis technology to meet the increasing demand of in vitro expression system and optimizes its transcription and translation system, so as to increase the yield of protein and provide a new way for its metabolism. There are still controversial arguments about translation strategies in transfer methods and interlingual methods [15].

\section{Deep Transfer Learning and the Process of Chinese-Korean Translation System}

2.1. Transfer Learning Diagram. As shown in Figure 1, transfer learning is divided into two parts: source domain and target domain. The process of transforming learning tasks into knowledge by source domain and knowledge into learning tasks by target domain is transfer learning [16]. Transfer learning also refers to the influence of one kind of learning on another kind of learning.

\subsection{Detailed Explanation of Deep Transfer Learning}

2.2.1. Deep Transfer Learning Classification. Deep transfer learning can be divided into four methods: transfer learning in case of instance, transfer learning in network fine-tuning, and deep transfer learning in confrontational and nonconfrontational domain adaptation. In use, the required deep transfer learning method can be selected according to the fine-tuning of examples and networks [17] and nonconfrontational and confrontational classification in Figure 2.

\subsubsection{Schema of Deep Transfer Learning under Fine} Adjustment. As shown in Figure 3, deep migration learning is divided into two parts: source domain and target domain, and the targets of source domain and target domain are finetuned [18]. The corresponding research cost is increased. However, after fine-tuning, the goal has more efficient and accurate results [19].

An important concept of deep learning is fine-tuning. This method is mainly to use the network that has been trained in the source domain, as shown in Figure 3, by freezing, and fine-tuning some network layers, adjusting for the target domain tasks, and obtaining the optimal network fine-tuning strategy, so as to achieve the target domain requirements.

\subsection{Establishment and Analysis of Translation System Model.} Language analysis is carried out through the user interaction interface, such as inputting the required translation, selecting the types of sentences, and finally displaying them at the front end [20]. The model interface includes multilingual decoding of multilingual encoder, generation of batch decoded data, and selection of language representation layer by labeling languages, so as to achieve the optimization result of simulation level. At the same time, the data 


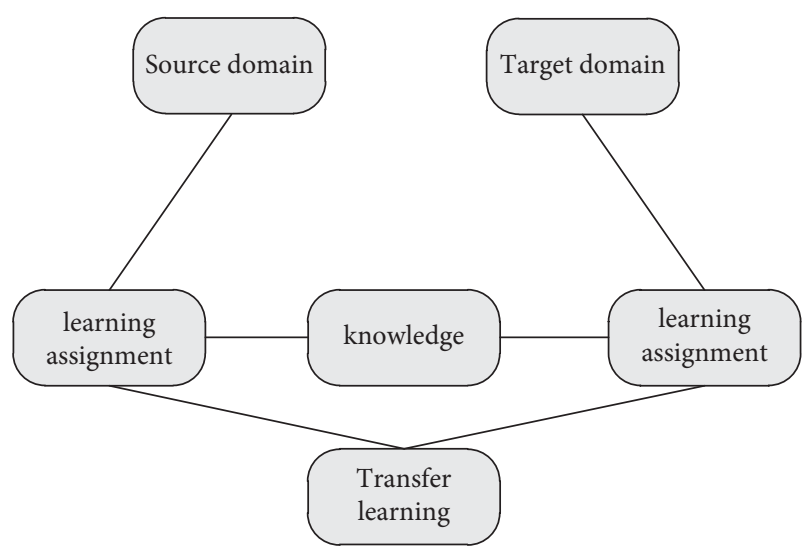

Figure 1: Transfer learning diagram.

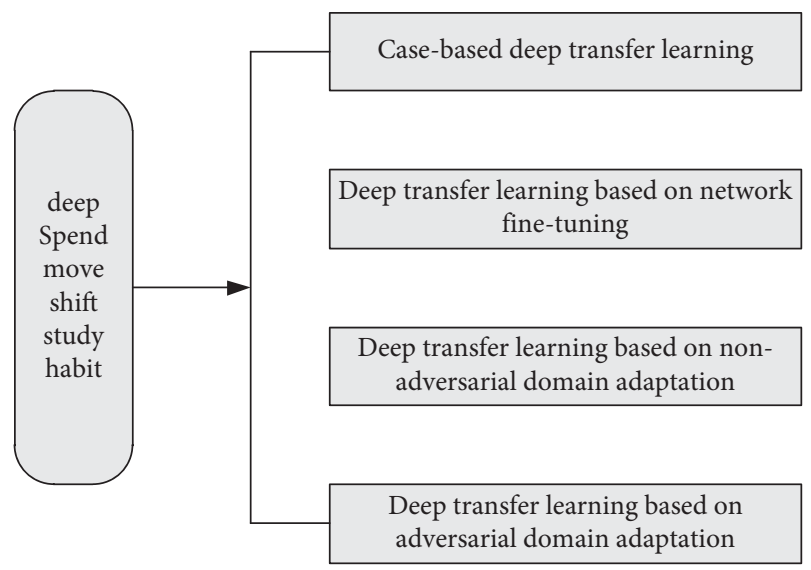

Figure 2: Deep transfer learning classification.

processing steps in this model include data cleaning, word segmentation regularization, and word segmentation. Data cleaning [21], word segmentation regularization, and word segmentation also achieve the functions of multilingual optimization in Figure 4.

2.4. Translation Process between China and Korea. The process of Chinese-Korean translation system is to input the Korean data to be translated first, then count the word frequency of the data, reduce the word frequency after the statistics are completed, and then reorganize the operation and train the relevant Korean data by using the BPE method. Finally, the related subwords are segmented to help more accurate translation [22], and the translation-related results are output in Figure 5.

\subsection{Comparison of Chinese and Korean Translation}

(1) It is a comparison of non-subject-predicate zero sentences in Chinese and Korean, which express interjection such as call, response, and question and answer in Table 1
(2) Interjection non-subject-predicate zero sentences expressing anger, dissatisfaction, and criticism in Table 2

(3) Interjection non-subject-predicate zero sentences expressing joy and happiness in Table 3

(4) Interjection non-subject-predicate zero sentences expressing surprise, sigh, and lament in Table 4

Through the comparison of non-subject-predicate zero sentences expressing anger, amazement, surprise, and joy in spoken Chinese and Korean, we can realize the differences in translation between Chinese and Korean.

\section{Deep Migration and Translation System}

\subsection{Deep Learning Model}

3.1.1. Definition of Deep Network Loss.

$$
l=l_{c}\left(D_{s}, y_{s}\right)+\lambda l_{A}\left(D_{s}, D_{t}\right),
$$

where $\ell$ represents target loss, $\ell_{c}$ represents classification loss, and $\lambda$ is a balance parameter.

\subsubsection{Deep Network Loss Composition.}

$$
L=L_{c}\left(D_{s}, y_{s}\right)+\lambda L_{D}\left(D_{s}, D_{T}\right),
$$

where $L_{\mathrm{c}}$ represents classified loss and $L_{\mathrm{D}}$ represents domain judgment loss [23].

3.1.3. MMD Metrics and Application Networks. MMD is the maximum mean difference. In formula (3), $H$ represents the regenerative Hilbert space with a feature core, $p, q$ represents two probability distributions, and $\varnothing()$ represents the nonlinear feature Yingshe function. Formula (4) represents the minimum batch source data and target domain data sampled from $S$ and $T$, respectively, and $K_{1}$ represents the core selected for layer $l$ of the deep neural network.

$$
\begin{aligned}
M M D_{H}(p, q) \triangleq & \left\|E_{p}\left[\phi\left(x_{i}^{s}\right)\right]-E_{q}\left[\phi\left(x_{i}^{t}\right)\right]\right\|_{H^{\prime}}^{2}, \\
M M D_{1}= & \frac{1}{n_{s}^{2}} \sum_{i=1}^{n_{s}} \sum_{j=1}^{n_{s}} K_{1}\left(\phi_{1}\left(X_{i}^{s}\right), \phi\left(X_{j}^{s}\right)\right) \\
& +\frac{1}{n_{t}^{2}} \sum_{i=1}^{n_{t}} \sum_{j=1}^{n_{s}} K_{1}\left(\phi_{1}\left(X_{i}^{t}\right), \phi_{1}\left(X_{j}^{t}\right)\right) \\
& -\frac{2}{n_{s} n_{t}} \sum_{i=1}^{n_{s}} \sum_{j=1}^{n_{t}} K_{1}\left(\phi_{1}\left(X_{i}^{s}\right), \phi_{1}\left(X_{j}^{t}\right)\right) .
\end{aligned}
$$

3.1.4. DDC Method

$$
l=l_{c}\left(D_{s}, y_{s}\right)+\lambda M M D^{2}\left(D_{s}, D_{t}\right) .
$$




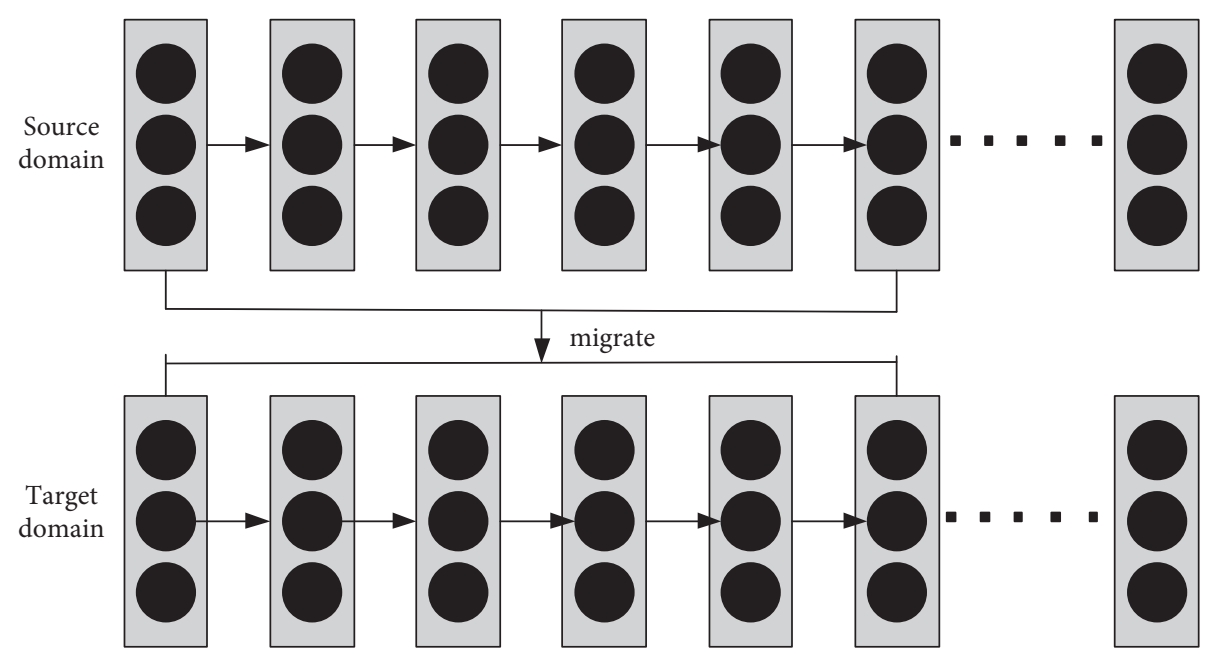

Figure 3: Diagram of deep transfer learning under fine-tuning.
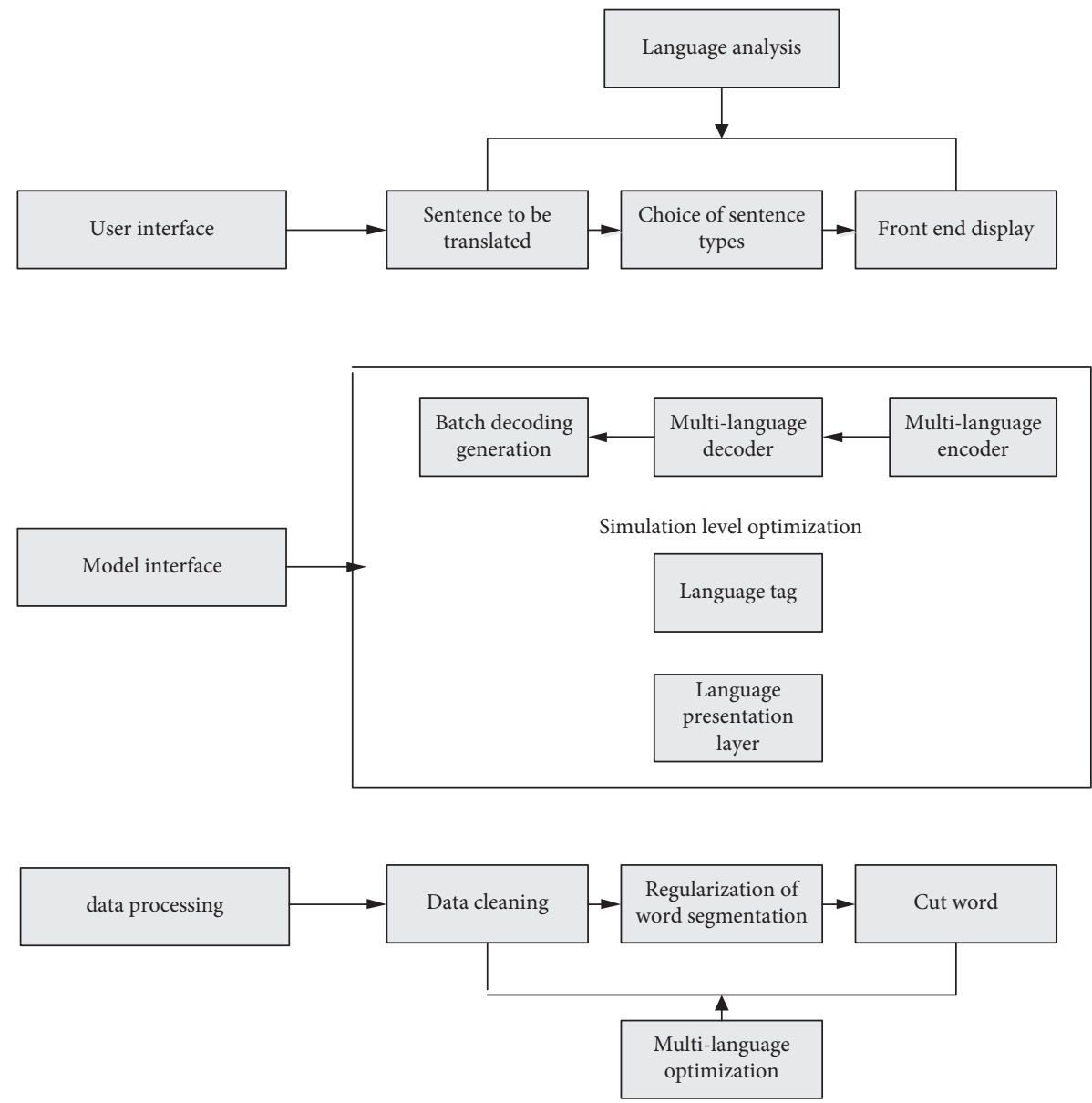

FIGURE 4: Establishment of the translation system model. 


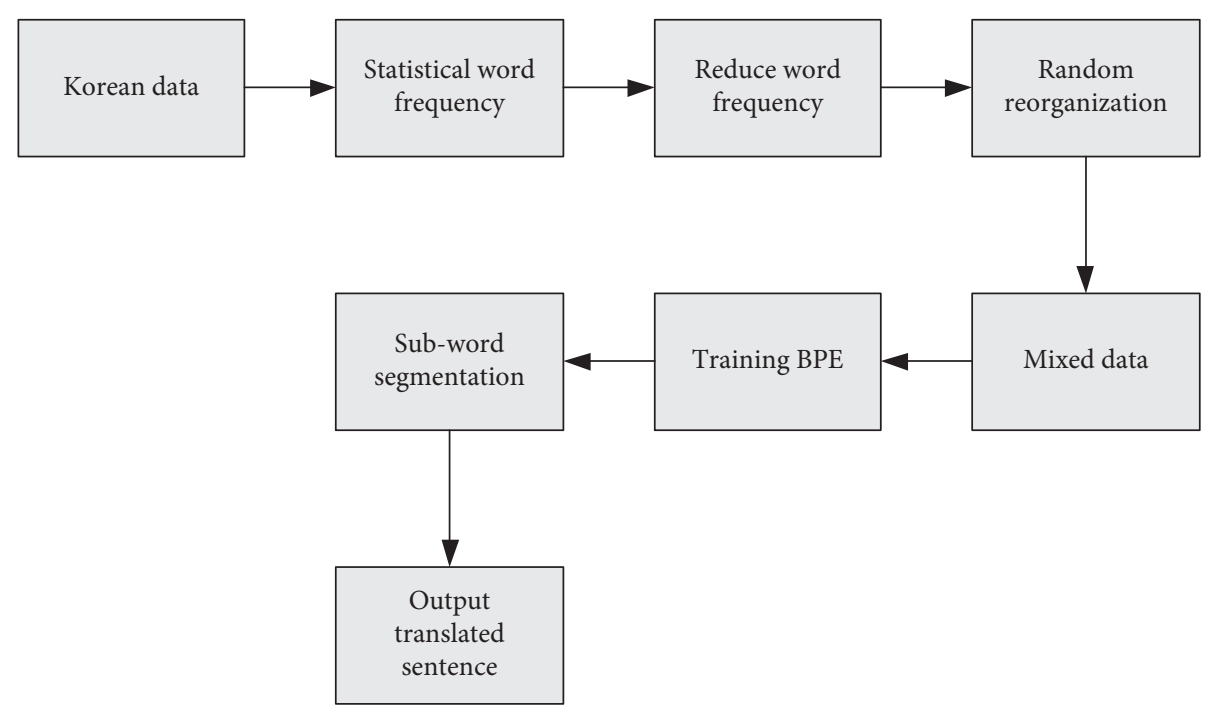

FIgURE 5: Chinese and Korean language translation process.

\subsubsection{Wasserstein Metric.}

$$
\begin{aligned}
W_{p}(f, g) & \stackrel{\operatorname{def}}{=}\left[\inf _{\gamma \in \Pi(f, g)} \int_{R^{d} \times R^{d}}\|x-y\|^{p} d \gamma(x, y)\right]^{(1 / p)}, \\
W_{1}(f, g) & =\sup _{\|f\|_{L \leq 1}} E_{x \sim f}[f(x)]-E_{y \sim g}[f(y)], \\
L_{w d D}\left(D_{j}\right) & =E_{x_{i}^{s j} \sim p_{s j}} D_{j}\left(F\left(x_{i}^{s j}\right)\right)-E_{x_{i}^{t} \sim p_{t}} D_{j}\left(F\left(x_{i}^{t}\right)\right),
\end{aligned}
$$

optimization formula : $L_{\mathrm{wdD}}(D)=\sum_{\mathrm{j}=1}^{N} L_{\mathrm{wdD}}\left(D_{j}\right)$,

gradient penalty expression : $L_{\text {grad }}\left(D_{j}\right)=\left(\left\|\nabla_{\hat{x}} D_{j}(\hat{x})\right\|_{2}-1\right)^{2}$,

maximum optimization training : $\max _{D_{j}} \sum_{\mathrm{j}=1}^{N} L_{w d_{D}}\left(D_{j}\right)-\beta L_{\text {grad }}\left(D_{j}\right)$.

WDGRL deep transfer learning loss function [24]:

3.1.6. Domain Distribution Similarity.

$$
\min _{\theta_{g}, \theta_{c}}\left\{\ell_{c}+\lambda \max _{\theta_{w}}\left[\ell_{w d}+\gamma \ell_{\mathrm{grad}}\right]\right\} \text {. }
$$

$$
\begin{aligned}
& \text { Objective function : } \min _{G, c} \sum_{i=1}^{N} L_{D_{i}}+\lambda \min _{G} M D^{2}\left(D_{s}, D_{T}\right), \\
& M D^{2}\left(D_{s}, D_{T}\right)=\sum_{K=1}^{2}\left(\frac{1}{N} \sum_{i=1}^{N}\left\|E\left(X_{i}^{k}\right)-E\left(X_{T}^{k}\right)\right\|_{2}+\left(\begin{array}{c}
N \\
2
\end{array}\right)^{-1} \sum_{i=1}^{N-1} \sum_{j=i+1}^{N}\left\|E\left(X_{i}^{k}\right)-E\left(X_{j}^{k}\right)\right\|_{2}\right) . \\
& L_{\text {total }}=L_{\mathrm{cls}}+\lambda L_{m m d}+\gamma L_{\text {disc }} .
\end{aligned}
$$

Single molecule heparin loss: 
TABLE 1: The comparison of Chinese and Korean statements such as response.

\begin{tabular}{ll}
\hline & Enjoy the non-subject-predicate zero sentence of part of speech \\
\hline \multirow{2}{*}{ Chinese } & 1. Express a call \\
& 2. Hey! Hi! Hey! Hey! \\
& 3. Indicate a response \\
& 4. Zhe! Mmm! Mmm! Mmm! \\
Korean & 5. Express a question and answer \\
& Huh? \\
\hline
\end{tabular}

TABle 2: Comparison of Chinese and Korean sentences such as anger.

\begin{tabular}{ll}
\hline & Enjoy the non-subject-predicate zero sentence of part of speech \\
\hline \multirow{3}{*}{ Chinese } & 1. Express anger, etc. \\
& 2. Yo! Hum! Bah! \\
& 3. Show contempt \\
& Yo! \\
\hline Korean & 1. Express anger, etc. \\
& 2. 음, 응! (Mm-hmm!)| 아, 그래(Hey) \\
& 3. Express criticism \\
& 글쎄! (Uh!) \\
\hline
\end{tabular}

TABLE 3: Comparison of Chinese and Korean sentences such as joy.

\begin{tabular}{ll}
\hline & Enjoy the non-subject-predicate zero sentence of part of speech \\
\hline Chinese & 1. Express happiness, happiness ha ha ha! \\
& 1. Express happiness, happiness \\
Korean & 하 하하 ! (Ha ha ha!)| \\
& 헤헤헤 ! (Hey hey hey!)| \\
& 2. Express a sigh \\
& 오!(Oh!)| 야!(Yah!) \\
\hline
\end{tabular}

TABLE 4: Comparison of Chinese and Korean sentences such as exclamation.

\begin{tabular}{ll}
\hline & Enjoy the non-subject-predicate zero sentence of part of speech \\
\hline & 1. Express surprise \\
Chinese & Ouch! Ah! \\
& Oh, my God! \\
& 2. Express lamentation \\
& Ouch! \\
\hline & 1. Express surprise \\
Korean & 아!(Ah!)| 아 Oㅣ참!(Cough!)| 오!(Oh!) \\
& 어머나!(Oh, my God!)| 아야!(Ouch!) \\
& 2. Express comprehension \\
& 맞다!(That’s right!) \\
\hline
\end{tabular}




\subsection{EEGNet Model}

\subsubsection{Convolution Calculation.}

$$
y=\sum_{\mathrm{i}=1}^{k_{1}} \sum_{\mathrm{j}=1}^{k_{2}} \sum_{\mathrm{l}=1}^{d} w_{\mathrm{ijl}} x_{\mathrm{ijl}}+\mathrm{b},
$$

where $W_{\mathrm{ijl}}$ is the weight and $y$ and $b$ are the offsets [25].

\subsubsection{Depth Separable Convolution.}

$$
\begin{array}{r}
\frac{D_{k} \times D_{k} \times \mathrm{M}+\mathrm{M} \times \mathrm{N}}{D_{k} \times D_{k} \times \mathrm{M} \times \mathrm{N}}=\frac{1}{N}+\frac{1}{D_{k} \times D_{k}}, \\
\frac{D_{k} \times D_{k} \times M \times D_{F} \times D_{F}+M \times N \times D_{F} \times D_{F}}{D_{k} \times D_{k} \times M \times N \times D_{F} \times D_{F}}=\frac{1}{N}+\frac{1}{D_{k} \times D_{k}} .
\end{array}
$$

\subsubsection{Softmax Model}

$$
P(i)=\frac{\exp \left(\theta_{i}^{T} x\right)}{\sum_{k=1}^{k} \exp \left(\theta_{k}^{T} x\right)} .
$$

\subsection{Machine Translation Model.}

$$
\begin{aligned}
h_{n} & =f\left(U \cdot x_{n}+W \cdot h_{n-1}+b\right), \\
c & =q\left(\left\{h_{1}, \ldots, h_{n}\right\}\right) .
\end{aligned}
$$

The probability $p$ of the resulting translation $y$ is

$$
\begin{aligned}
P(y) & =\prod_{t=1}^{T} p\left(y_{t} \mid\left\{y_{1}, \ldots, y_{t-1}\right\}, c\right), \\
p\left(y_{t} \mid\left\{y_{1}, \ldots, y_{t-1}\right\}, c\right) & =g\left(y_{t-1}, s_{t}, c\right), \\
c_{t} & =\sum_{j=1}^{n} \alpha_{t, j} h_{j}, \\
\alpha_{t, j} & =\frac{e^{e_{t, j}}}{\sum_{k=1}^{n} e^{e_{t, j}}}, e_{t . j}=a\left(s_{t-1}, h_{t}\right) .
\end{aligned}
$$

\section{Deep Transfer Learning and the Design and Analysis of Chinese-Korean Translation System}

\subsection{Deep Transfer Learning Model}

4.1.1. Accuracy Analysis of Different Adjustment Mechanisms Based on Different MMD Measures. As shown in Table 5, the dataset is divided into three parts: dataset 1 , dataset 2 , and average, and the accuracy of datasets 1 and 2 under different adjustment mechanisms under MMD measurement is investigated. Among them, the accuracy of dataset 1 is $62.7 \%$ without weight adjustment mechanism, $68.9 \%$ under single source weight, and $67.1 \%$ under multiple weights. Under the weightlessness mechanism, the average value is $59.0 \%$, the single source weight is $64.6 \%$, and the multisource weight is $75.1 \%$. Through three sets of data, we can see that the accuracy under MMD measurement is lower under no weight adjustment mechanism and higher under multisource weight and the overall accuracy is on the rise. Among them, the correct rate of dataset 1 under different adjustment mechanisms is above the average correct rate.

In order to make the accuracy trend more intuitive, the following chart is drawn. As shown in Figure 6, the accuracy rate of datasets 1 and 2 is the highest under the multisource weight adjustment mechanism, while the accuracy rate of datasets 1 and 2 is lower under the nonweight adjustment mechanism. The accuracy of dataset 1 in different adjustment mechanisms under MMD measurement is generally higher than the average.

4.1.2. Accuracy Analysis of Different Adjustment Mechanisms Based on Different Wasserstein Measures. Through the data values 1 and 2 and the average value, the accuracy of data under different adjustment mechanisms under Wasserstein metric is discussed. The accuracy of dataset 1 is $68.5 \%$ under no weighting mechanism, $72.8 \%$ under single source weighting mechanism, and $82.5 \%$ under multisource weighting mechanism. The accuracy rate of dataset 2 is $55.4 \%$ under no weight adjustment mechanism, 59.8\% under single source weight, and $66.8 \%$ under multisource weight. The average accuracy rate is $61.2 \%$ under no weight, $66.7 \%$ under single source weight, and $73.2 \%$ under multisource weight. Among them, the accuracy of dataset 1 is higher under different adjustment mechanisms in Table 6.

It can be seen from Figure 7 that the correct rate of datasets 1 and 2 and the average value is on the rise under the adjustment mechanism of weightlessness, single source weight, and multisource weight, while the correct rate of weightlessness mechanism is lower and the correct rate of multisource weight is higher. Also, the accuracy rate of dataset 1 is higher than that of dataset 2 and the average value. Combined with the chart under MMD metric, we can see that the accuracy rate under multisource weight is higher than that under no weight and the accuracy rate of multisource weight in dataset 1 under Wasserstein metric is $82.5 \%$ which is lower than that under MMD metric (83.1\%).

4.1.3. Comparative Analysis. Comparing the Wasserstein model with the MMD without weight, the average accuracy of MMD without weight was $59 \%$, the average accuracy under multisource weight was $61.2 \%$, the average accuracy under single source weight was $64.6 \%$ and $66.7 \%$, and the average accuracy under multisource weight was $75.1 \%$ and $73.2 \%$, respectively. Through data analysis, it is learned that the accuracy rate of Wasserstein model is higher than MMD under no weight and single source weight and should be preferred, but the correct rate of MMD model is higher under multisource weight.

4.2. Nonlinear Activation Function of Deep Transfer Learning. Based on the analysis of inactive function under deep transfer learning, ReLU function has countless values after 
TABLE 5: Accuracy analysis of MMD regulation mechanism.

\begin{tabular}{lccc}
\hline Dataset & No weight (\%) & Single source weight (\%) & Multisource weight (\%) \\
\hline Dataset 1 & 62.7 & 68.9 & 83.1 \\
Dataset 2 & 55.3 & 60.2 & 67.1 \\
Average & 59.0 & 64.6 & 75.1 \\
\hline
\end{tabular}

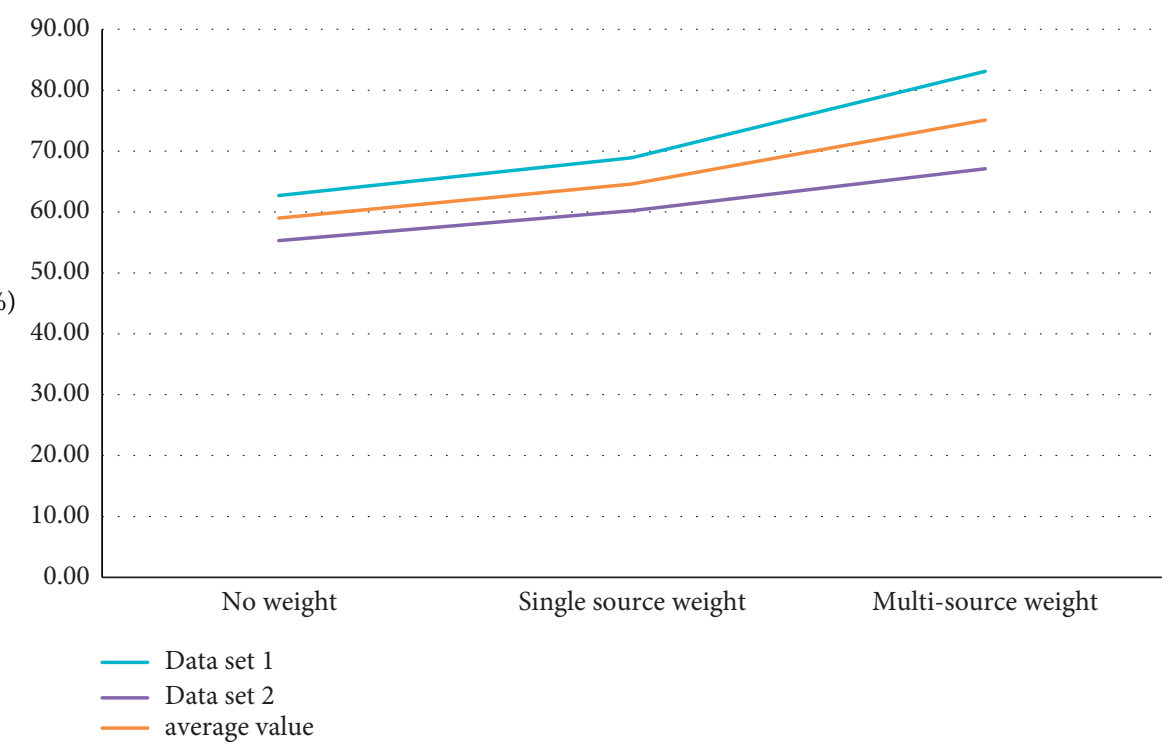

Figure 6: Accuracy of different mechanisms under MMD measurement.

TABLE 6: Accuracy analysis of Wasserstein regulation mechanism.

\begin{tabular}{lccc}
\hline Dataset & No weight (\%) & Single source weight (\%) & Multisource weight (\%) \\
\hline Dataset 1 & 68.5 & 72.8 & 82.5 \\
Dataset 2 & 55.4 & 59.8 & 66.8 \\
Average & 61.2 & 66.7 & 73.2 \\
\hline
\end{tabular}

(\%)

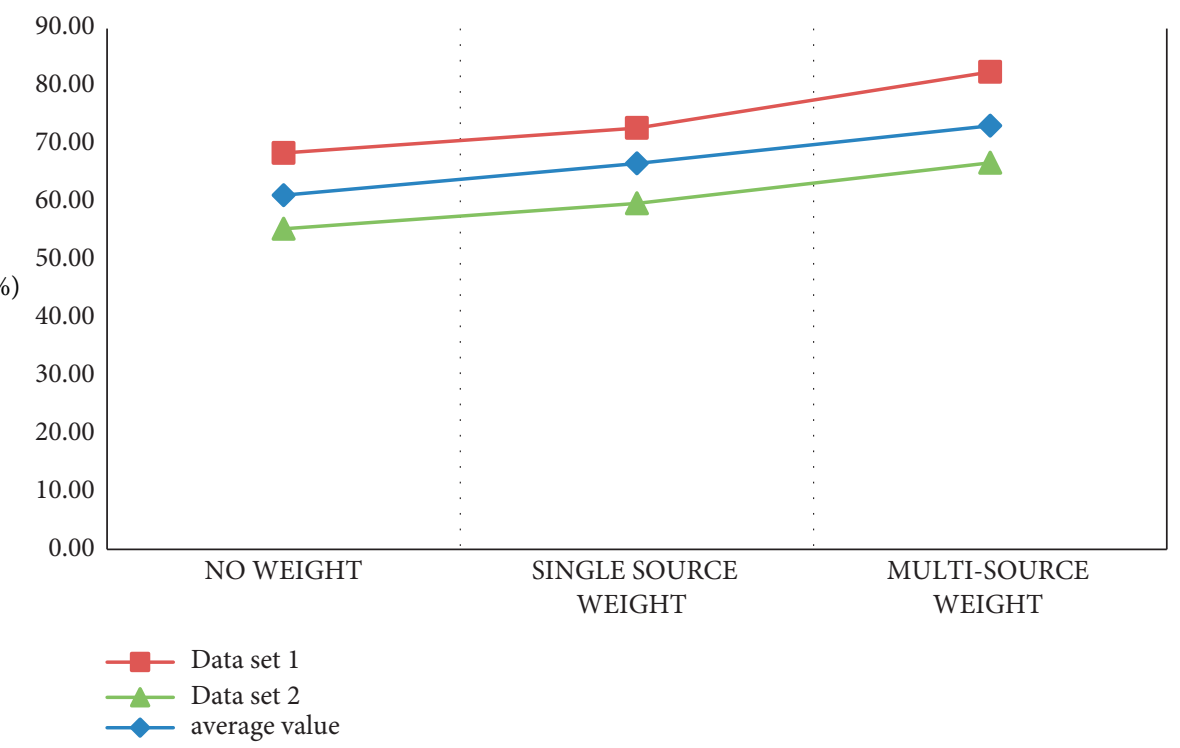

Figure 7: Accuracy of different mechanisms under Wasserstein metric. 
zero and shows an upward trend after zero. The sigmoid function image shows a slow upward trend after zero point and approaches the value 1 when $x$ is about 5 , and the value infinitely approaches 1 when $x$ is about 10 . Tanh's function is -1 before zero, rises slowly after zero, and finally approaches the value of 1 infinitely in Figure 8.

ReLU Function. Pros: fast convergence speed, avoid gradient disappearance, and simple calculation. Cons: no boundaries.

Sigmoid Function. Pros: from the image point of view: is a continuous function and easy to guide; mathematically: there is a good spatial mapping effect; functional point of view: a number into a popular sense of grasp representation. Cons: the gradient disappears when it is propagated in reverse.

Tanh Function. Pros: has all the advantages of sigmoid function. Cons: gradients disappear and $\exp ()$ calculations are expensive.

4.3. Comparison of EEGNet Method Models. It can be seen from Figure 9 that the correct rate of EEGNet_0 model is about $59 \%$, the correct rate of EEGNet_1 model is $66.5 \%$, and the correct rate of EEGNet_2 model is about $62.3 \%$. Through data analysis, we can see that the correct rate of EEGNet_1 model is higher than that in EEGNet_0 and EEGNet_2. Its overall average law is also higher than $50 \%$. The effect of EEGNet_1 was higher than that of EEGNet_0 and EEGNet_2.

4.4. Analysis of Chinese and Korean Translation System Model. According to the comparison of $\mathrm{N}$-grams and EL-grams translation models, the correct rate of $\mathrm{N}$-grams translation into Korean is $75.1 \%$ and the correct rate of EL-grams translation into Chinese is $89.43 \%$. The perplexity of translating into Korean is $126.39 \%$, while the perplexity of translating into Chinese is $34.54 \%$. In the EL-grams model, the correct rate of translation into Korean is $80.34 \%$ and the correct rate of translation into Chinese is $93.21 \%$. The perplexity of translating into Korean is $105.33 \%$ and that of Chinese is $18.88 \%$. By comparison, we can see that the accuracy of N-grams model is higher than that of EL-grams model in Chinese and Korean translation models and the confusion of $\mathrm{N}$-grams model is lower than that of EL-grams model. Therefore, the performance of N-grams model is better than that of EL-grams system (Table 7).

It can be seen from Figure 10 that the accuracy of $\mathrm{N}$-grams Chinese-Korean translation system model is higher than that of EL-grams model, but its confusion is lower than that of EL-grams translation model system. Therefore, the performance of EL-grams translation model is lower than that of N-grams translation model. In order to ensure the accuracy of translation, the $\mathrm{N}$-grams Chinese-Korean translation system model should be given priority when choosing translation system.

4.5. Investigation on the Difficulty of Korean Translation. According to the survey, most people think that the translation between China and South Korea is difficult, accounting for $48 \%$, and only $3 \%$ think it is very easy. It shows that there are some difficulties in Chinese-Korean translation. Through this Chinese-Korean translation system, the translation difficulty is reduced and the translation efficiency is improved (Figure 11).

Through data and graph analysis, we know that the vast majority of the people surveyed think that Chinese and Korean translation is more difficult, but now, trade, travel, etc. are inseparable from Chinese and Korean translation, so it is necessary to design a high-efficiency, high-performance Chinese-Korean translation system.

\subsection{System Test}

4.6.1. Performance Test of Three Methods. The related system tests are carried out. Based on Table 8, it can be seen that, in the Chinese-Korean translation system based on blockchain, when the translation sentences are 100 sentences, the average response time is $1.230 \mathrm{~ms}$ and the peak traffic response time is $1.556 \mathrm{~ms}$; when 500 sentences are translated, the average response time is $1.556 \mathrm{~ms}$ and the peak traffic response time is $1.890 \mathrm{~ms}$. When 1000 sentences are translated, the average response time is $2.098 \mathrm{~ms}$ and the peak traffic response time is $2.121 \mathrm{~ms}$. According to the performance tests of 100 sentences, 500 sentences, and 1000 sentences, the performance tests are all passed, which proves that this method is feasible.

Based on the performance test of the Chinese-Korean translation system proposed in this paper, the average response time and peak traffic response time are $1.980 \mathrm{~ms}$ and $2.021 \mathrm{~ms}$, respectively, when the translation sentences are 100 sentences. When 500 sentences are translated, the average response time and peak traffic response time are $2.99 \mathrm{~ms}$ and $3.005 \mathrm{~ms}$, respectively. When 1000 sentences are translated, the average response time and peak traffic response time are $4.236 \mathrm{~ms}$ and $4.653 \mathrm{~ms}$, respectively. The performance test results are all passed, and the method proposed in this paper is feasible (Table 9).

According to the analysis of traditional Chinese and Korean translation system methods, the average response time of translated sentences with 100,500 , and 1000 sentences is higher than that of the method proposed in this paper and the peak traffic response time is also higher than that of the translation system proposed in this paper and the blockchain translation system. However, the performance test of its translation system has passed (Table 10).

From Figure 12, it can be seen that the performance test results of the Chinese-Korean translation system proposed in this paper are higher than those of the traditional ChineseKorean translation system, and the data of the ChineseKorean translation system in blockchain are close, indicating that the Chinese-Korean translation system proposed in this paper can meet the daily Chinese-Korean translation needs and its performance is excellent. The Chinese-Korean translation system is feasible.

The Chinese-Korean translation system used in this paper passes the system test, and the test results are shorter and more efficient than the traditional Chinese-Korean 

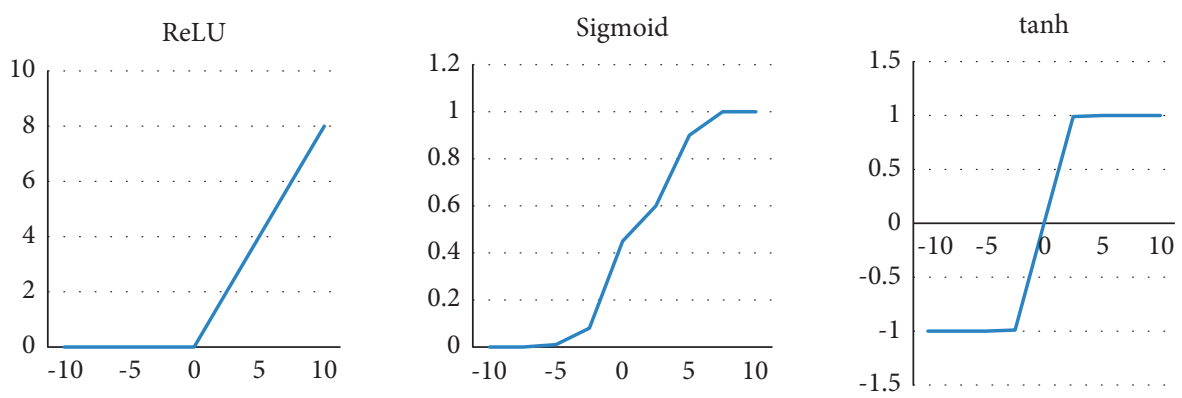

FiguRE 8: Deep transfer learning inactive function.

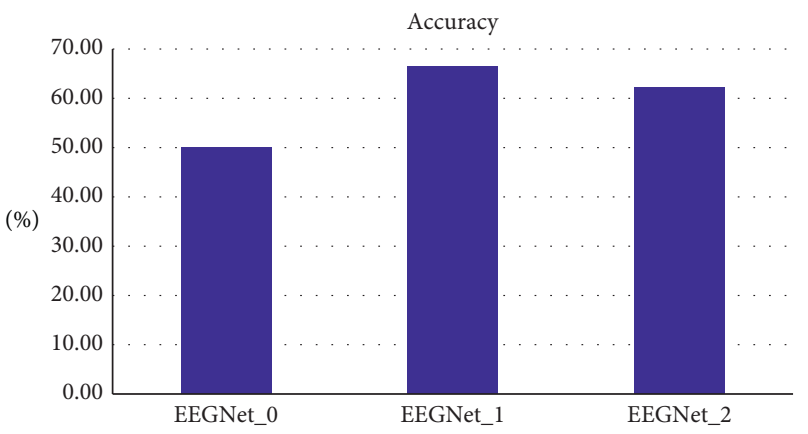

Figure 9: Comparison of EEGNet models.

TABle 7: Comparative analysis of different translation models.

\begin{tabular}{lcc}
\hline Model & PPL $_{\text {Korean }} ;$ accuracy & PPLean $_{\text {Chinese; }}$ accuracy \\
N-grams & $126.39 ; 75.18$ & $34.54 ; 89.43$ \\
EL-grams & $105.33 ; 80.34$ & $18.88 ; 93.21$ \\
\hline
\end{tabular}

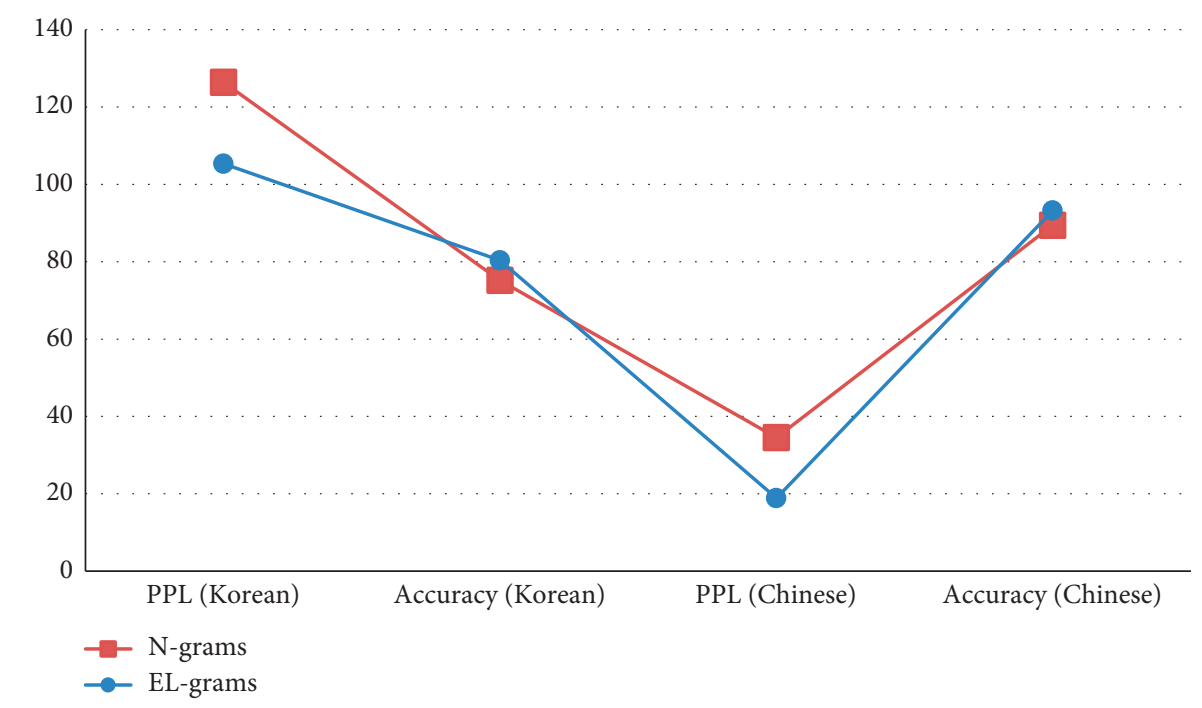

FIgURE 10: N-grams and EL-grams model testing. 


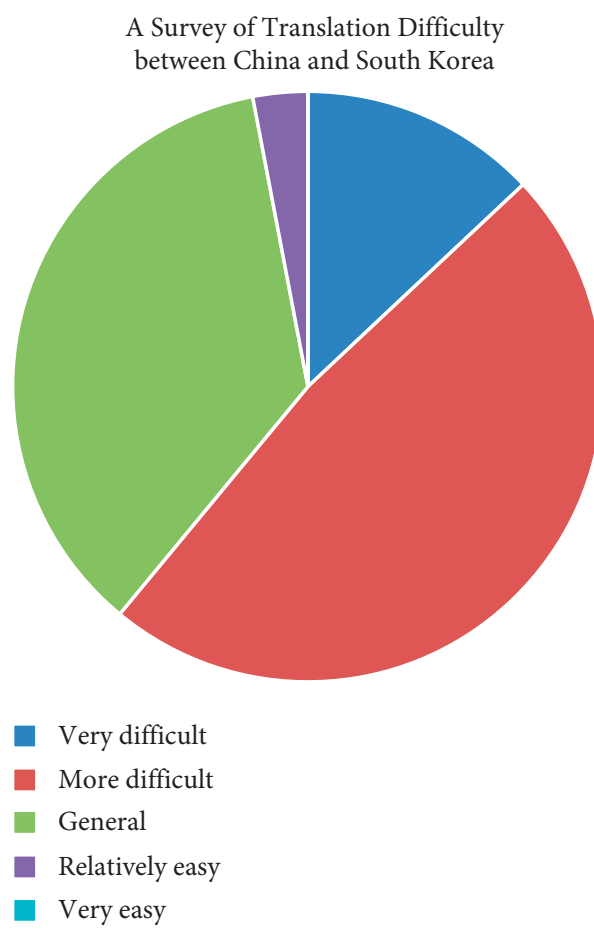

Figure 11: Survey of translation difficulty between Chinese and Korean.

TABLE 8: Performance test of Chinese and Korean translation system based on blockchain.

\begin{tabular}{lccc}
\hline Translated sentence & Average response time $(\mathrm{ms})$ & Peak flow response time & Test conclusion \\
\hline 100 & 1.230 & 1.556 & Pass \\
500 & 1.556 & 1.890 & Pass \\
1000 & 2.098 & 2.121 & Pass \\
\hline
\end{tabular}

TABle 9: Performance test of Chinese and Korean translation system based on this method.

\begin{tabular}{lccc}
\hline Translated sentence & Average response time $(\mathrm{ms})$ & Peak flow response time & Test conclusion \\
\hline 100 & 1.980 & 2.021 & Pass \\
500 & 2.997 & 3.005 & Pass \\
1000 & 4.236 & 4.653 & Pass \\
\hline
\end{tabular}

TABle 10: Performance test of Chinese-Korean translation system based on traditional methods.

\begin{tabular}{lccc}
\hline Translated sentence & Average response time $(\mathrm{ms})$ & Peak flow response time & Test conclusion \\
\hline 100 & 1.988 & 3.210 & Pass \\
500 & 3.497 & 6.334 & Pass \\
1000 & 4.936 & 7.203 & Pass \\
\hline
\end{tabular}

translation system. When the translation statement is 1000 , the average response time of the Chinese-Korean translation system proposed in this paper is $4.236 \mathrm{~ms}$, while that of the traditional translation system is $4.936 \mathrm{~ms}$. In contrast, the Chinese and Korean translation systems perform better.

4.6.2. Performance Test Analysis. It is divided into the following groups of data: 500, 1000, 1500, 2000, 2500, and 3000.
There are seven sets of data, and the specific analysis is shown in the following table. According to the comparison of blockchain system method, Chinese and Korean translation system, and traditional translation system, the average time of blockchain method and this translation method is close, both within $13 \mathrm{~ms}$, and the success rate is $100 \%$, while the average time of traditional translation method exceeds $20 \mathrm{~ms}$. The success rate of blockchain method is $100 \%$, the highest success rate of this translation system is $100 \%$, and 


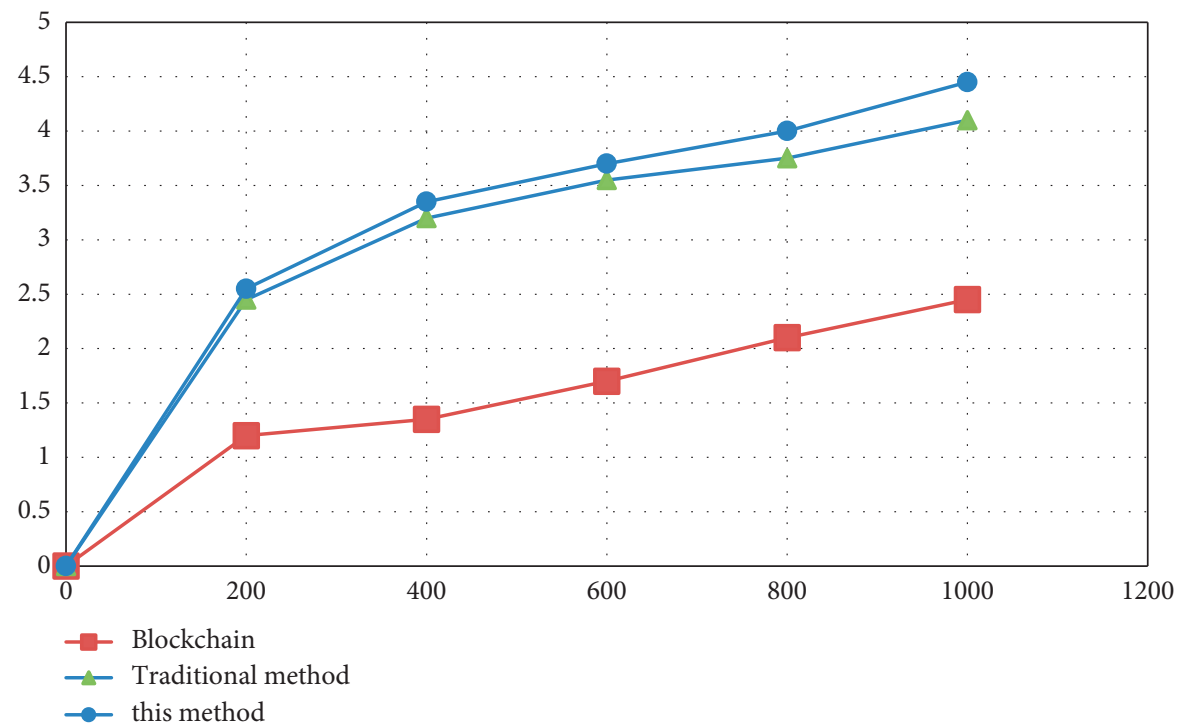

FIGURE 12: Comparative analysis of average response time.

TABLE 11: System test structure.

\begin{tabular}{|c|c|c|c|c|c|c|c|}
\hline System & Number of query requests & 500 & 1000 & 1500 & 2000 & 2500 & 3000 \\
\hline \multirow{2}{*}{ Blockchain method } & Mean response time (MS) & 10 & 23 & 33 & 58 & 73 & 97 \\
\hline & Success rate $(\%)$ & 100 & 100 & 100 & 100 & 100 & 100 \\
\hline \multirow{2}{*}{ The Chinese-Korean translation system } & Mean response time (MS) & 13 & 24 & 38 & 59 & 75 & 99 \\
\hline & Success rate $(\%)$ & 100 & 99.8 & 99.7 & 99.64 & 99.75 & 99.6 \\
\hline \multirow{2}{*}{ Traditional translation system } & Mean response time (MS) & 21 & 42 & 59 & 83 & 101 & 134 \\
\hline & Success rate $(\%)$ & 100 & 99.3 & 98.3 & 97.8 & 99.3 & 99 \\
\hline
\end{tabular}

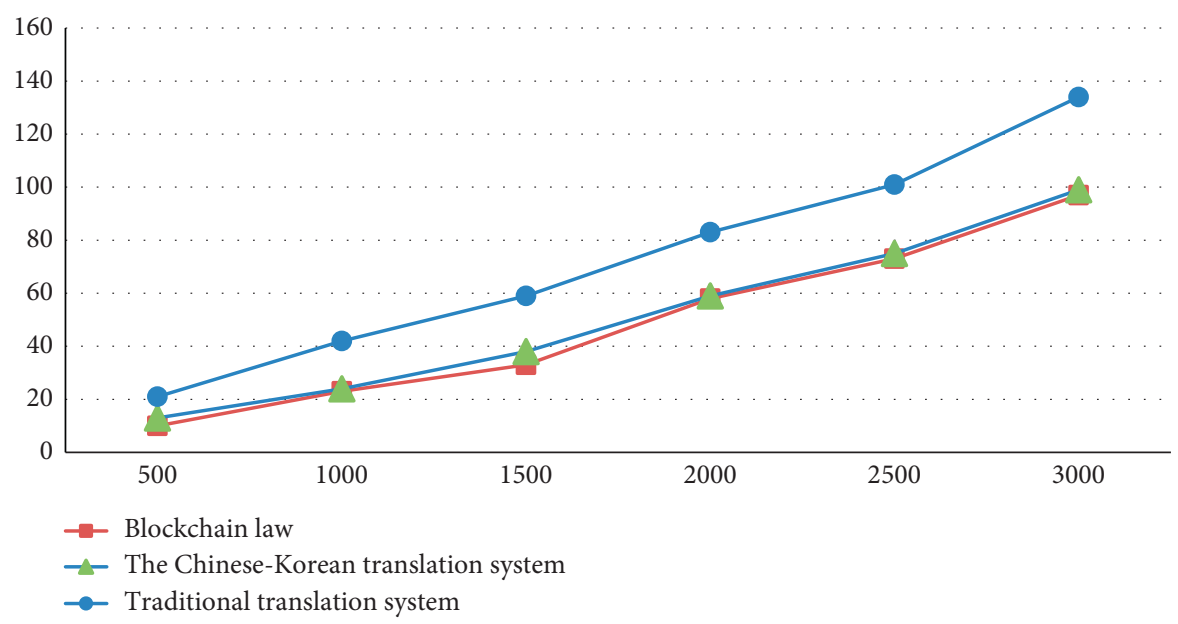

FIGURE 13: Time performance test analysis of translation system.

the lowest success rate is $99.6 \%$ when the data are 3000 . The highest accuracy rate of traditional translation method is $100 \%$, and the lowest accuracy rate is $99 \%$ when the data are 3000. According to the data, the accuracy of this translation method is higher than that of the traditional translation system and the average response time is slightly lower than that of the traditional translation system (Table 11).
It can be seen from Figure 13 that the average response time of the proposed Chinese-Korean translation system is lower than that of the traditional translation system, which saves translation time and improves translation efficiency. This system can meet the needs of normal translation.

From Figure 14, we can see that the correct rate of Chinese and Korean translation system is higher than that of 


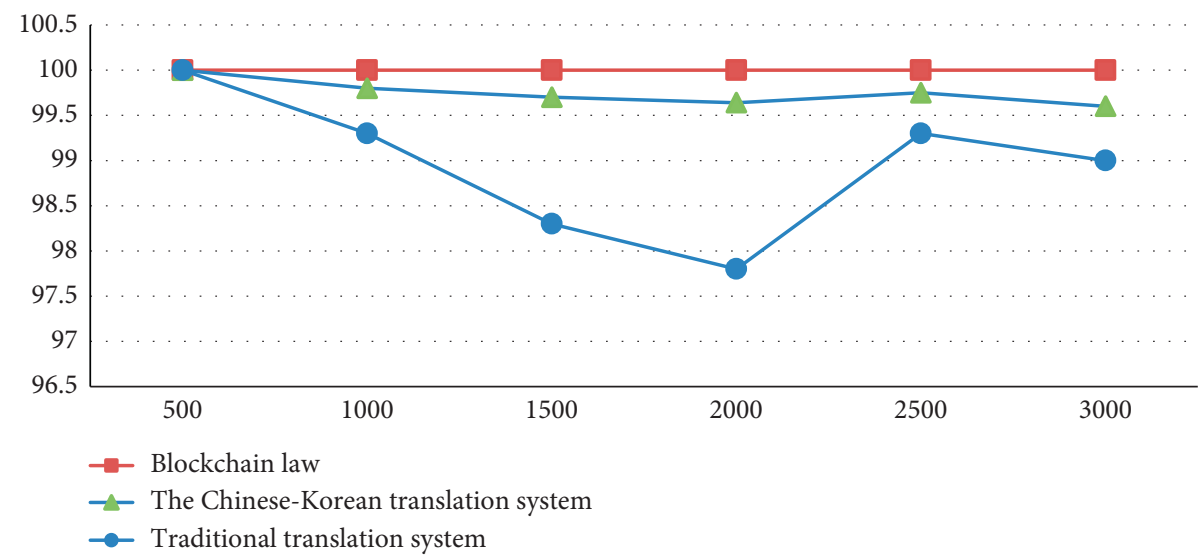

Figure 14: Performance test analysis of translation system accuracy rate.

traditional translation system, which is close to $100 \%$. Also, the accuracy rate fluctuates little when the data increase.

\section{Conclusion}

Through the analysis of the loss composition of deep migration and the accuracy under different adjustment mechanisms under MMD and Wasserstein metrics, this paper discusses and analyzes the requirements of maximizing the efficiency and accuracy of the Chinese-Korean translation system. By systematically testing the ChineseKorean translation system model, blockchain model, and traditional translation system, it can be seen that the Chinese-Korean translation model meets the daily translation requirements. All the tests are passed, and the accuracy and efficiency are higher than those of the traditional translation system, thus improving the accuracy and efficiency of the Chinese-Korean translation system. In real life, it is inevitable to communicate with different people, so in order to solve the language problem, the Chinese-Korean translation system reflects its value. In the process of future research, it should be optimized with a variety of translation models to improve the accuracy and efficiency of translation system.

\section{Data Availability}

The experimental data used to support the findings of this study are available from the corresponding author upon request.

\section{Disclosure}

This is the current achievement of "Parallel Corpus-Based Hybrid Translation Teaching Reform and Practice," a teaching reform project in 2019 by The Education Department of Liaoning Province.

\section{Conflicts of Interest}

The authors declare that they have no conflicts of interest regarding this work.

\section{References}

[1] J. Yoo, S. Sabir, and D. Heo, "Deep learning can reverse photon migration for diffuse optical tomography," IEEE Transactions on Medical Imaging, vol. 23, no. 5, pp. 23-45, 2017.

[2] C. Sun, Y. Yang, C. Wen, K. Xie, and F. Wen, "Voiceprint identification for limited dataset using the deep migration Hybrid model based on transfer learning," Sensors, vol. 18, no. 7, Article ID 2399, 2018.

[3] P. Sun, J. Lan, J. Li, Z. Guo, Y. Hu, and T. Hu, "Efficient flow migration for NFV with Graph-aware deep reinforcement learning," Computer Networks, vol. 183, no. 4, Article ID 107575, 2020.

[4] K. Cui, C. Hu, R. Wang, Y. Su, H. Mao, and H. Li, "Deeplearning-based extraction of the animal migration patterns from weather radar images," Sciece China. Information Sciences, vol. 63, no. 4, Article ID 140304, 2020.

[5] Q. Tong, L. I. Taijun, and X. Liu, "Vehicle information recognition method based on deep migration learning," Video Engineering, vol. 55, no. 8, pp. 54-75, 2019.

[6] M. Avila, L. N. Osorio, J. Fernandes et al., "Migration deconvolution via deep learning," Pure and Applied Geophysics, vol. 178, no. 2, 2021.

[7] J. Sun, X. He, X. Ge, X. Wu, J. Shen, and Y. Song, "Detection of key organs in tomato based on deep migration learning in a complex background," Agriculture, vol. 8, no. 12, 2018.

[8] M. Liu, X. Wang, and L. Zhou, "Study on extraction and recognition of traditional Chinese medicine tongue manifestation: Based on deep learning and migration learning," Journal of Traditional Chinese Medicine, vol. 34, no. 7, pp. $67-89,2019$.

[9] W. Zhang and J. Gao, "Deep-learning full-waveform inversion using seismic migration images," IEEE Transactions on Geoscience and Remote Sensing, vol. 60, no. 99, pp. 1-18, 2021.

[10] P. Xiao, G. Zhou, and M. He, "Apple image recognition based on deep and migration learning," International Agricultural Engineering Journal, vol. 27, no. 3, pp. 371-379, 2018.

[11] H. Chalupsky, "OntoMorph: a translation system for symbolic knowledge," Principles of Knowledge Representation \& Reasoning, vol. 45, no. 12, pp. 66-87, 2000.

[12] M. Johnson, M. Schuster, Q. V. Le et al., "Google's multilingual neural machine translation system: Enabling zero-shot translation," Transactions of the Association for Computational Linguistics, vol. 5, no. 2, pp. 109-112, 2016. 
[13] R. D. Brown, "Adding linguistic knowledge to a lexical example-based translation system," Tmi, vol. 7, no. 3, pp. 55-76, 1999.

[14] F. Caschera and V. Noireaux, "Synthesis of $2.3 \mathrm{mg} / \mathrm{ml}$ of protein with an all Escherichia coli cell-free transcriptiontranslation system," Biochimie, vol. 99, pp. 162-168, 2014.

[15] M. Kay and J. M. Gawron, "Verbmobih a translation system for face-to-face dialog," Csli Lecture Notes, vol. 87, no. 23, pp. 134-156, 1994.

[16] M. Duggan, J. Duggan, E. Howley, and E. Barrett, “A reinforcement learning approach for the scheduling of live migration from under utilised hosts," Memetic Computing, vol. 65 , no. 23 , pp. 178-196, 2017.

[17] L. Morrice, "The learning migration nexus: Towards a conceptual understanding," European Journal for Research on the Education and Learning of Adults, vol. 5, no. 2, pp. 149-159, 2014.

[18] K. Monkman, "Transnational migration and learning processes of Mexican adults constructing lives in California," International Journal of Educational Development, vol. 19, no. 4-5, pp. 367-382, 1999.

[19] K. Caskey and B. Subirana, "Supporting SME e-commerce migration through blended e-learning," Journal of Small Business and Enterprise Development, vol. 14, no. 4, pp. 670-688, 2007.

[20] M. Arif, A. Kiani, and J. Qadir, "Machine learning based optimized live virtual machine migration over WAN links," Telecommunication Systems: Modeling, Analysis, Design and Management, vol. 34, no. 21, pp. 56-78, 2017.

[21] V. A. . Kolb, E. V. Makeyev, and A. S. Spirin, "Co-translational folding of an eukaryotic multidomain protein in a prokaryotic translation system." Journal of Biological Chemistry, vol. 275, no. 22 , pp. 16597-16601, 2000.

[22] S. Nakamura, K. Markov, H. Nakaiwa et al., "The ATR multilingual speech-to-speech translation system," IEEE Transactions on Audio Speech and Language Processing, vol. 14, no. 2, pp. 365-376, 2006.

[23] Y. Chadani, K. Ono, S. I. Ozawa, and Y. Takahashi, "Ribosome rescue by Escherichia coli ArfA (YhdL) in the absence of transtranslation system." Molecular Microbiology, vol. 78, no. 4, pp. 796-808, 2010.

[24] B. S. Negrutskii, R. Stapulionis, and M. P. Deutscher, "Supramolecular organization of the mammalian translation system," Proceedings of the National Academy of Sciences, vol. 91, no. 3, pp. 964-968, 1994.

[25] J. M. . Betton, "Rapid translation system (RTS): A promising alternative for recombinant protein production," Current Protein \& Peptide Science, vol. 4, no. 1, pp. 73-80, 2003. 